\title{
Short Circuit Detection in Stator Winding Of Three Phase Induction Motor Using Wavelet Transform and Quadratic Discriminant Analysis
}

\author{
D.A. Asfani ${ }^{\mathrm{a}^{*}}$, I Made Yulistya Negara ${ }^{\mathrm{a}}$, P. Perdana Surya ${ }^{\mathrm{a}}$ \\ ${ }^{a}$ Electrical Engineering Department, Institut Teknologi Sepuluh Nopember, \\ Jl. Arief Rahman Hakim, Surabaya 60111, Indonesia \\ *Corresponding Author: anton@ee.its.ac.id
}

\begin{abstract}
Wavelet transform has become popular because of satisfied result for identifying short circuit in stator of induction motor. This paper presents prototype of discrete wavelet transform combined with quadratic discriminant analysis for monitoring of induction motor condition. Mode of motor operation is introduced in this paper as normal operation, starting of fault, and ending of fault. For this task, Discrete wavelet transform with Quadratic discriminant analysis is used to classify motor current into this three state of motor operation. Motor current is processed by discrete wavelet transform with Haar filter to extract high frequency of signal. Then, energy level calculated from high frequency signal is evaluated with quadratic discriminant analysis to identifying states of motor current condition.
\end{abstract}

Keywords: Induction motor winding, Quadratic discriminant analysis, Wavelet transforms.

\section{Introduction}

Induction motors are a critical component of most of industrial processes and are always integrated with production equipment. Induction motor is widely used because it has strong construction, high efficiency, and cheap maintenance. Stopping of induction motor would cause serious problem and huge cost losses.

Long time operation, wet environment and frequently vibration could make this motor's condition dangerous and got serious damage. Research of fault in induction motor which has been done shows us fault mechanism of induction motor categorized as fault of bearing $(41 \%)$, fault of stator $(37 \%)$, fault of rotor $(10 \%)$ and others $(12 \%)^{[1]}$.

Because of development in digital signal processing technology, information that is not able to get from some collecting data have been able to extracted. In the light of these developments, some methods for monitoring of induction motor have been invented and introduced since many years ago. Mechanical-vibration analysis ${ }^{[1]}$, and also by thermal behavior, infrared recognition, radio-frequency emissions, and chemical analysis is used to detect fault in induction motor, but it is second order effects when any fault occurred in induction motor if we compared them to stator current variation. Moreover, if we used vibration analysis method, it will be affected by other vibration from other induction motor. Furthermore, in a number of cases, the severity of the fault (for example, number of broken rotor bars) must be high to make it detectable by the above-specified methods ${ }^{[2]}$. Then, condition monitoring via MCSA (Motor Current Signature Analysis) has recently attracted more attention from researchers and also has been widely used to diagnose problems in electrical motors ${ }^{[3]-[12]}$. MCSA focuses its work on spectral analysis of stator current and has been satisfy applied to detect broken rotor bars ${ }^{[3-4]}$, abnormality of air gap eccentricity ${ }^{[5]}$, and shorted inter-turns in stator windings ${ }^{[6]}$, among other mechanical problems ${ }^{[7-9]}$. In addition, many industrial cases have been analyzed by MCSA since $1980 \mathrm{~s}^{[10-12]}$ with good results ${ }^{[12]}$.

Stator current of induction motor is analyzed by MCSA in search of current harmonics directly related to new rotating flux components caused by faults in the motor-flux distribution ${ }^{[2]}$. The advantage of this technique is that it is realized nowadays as a standard because its simplicity: It needs only one current sensor per machine. Furthermore, Fast Fourier Transforms (FFT) as principle in MCSA is very applicable.

FFT is suitable applied in stationary or continuous signal processing. Nevertheless, it will be serious problem if motor current as input in MCSA is non-stationary signal. Changing time domain to frequency domain using FFT will 
result to loss time information in current signal. So, when looking at FFT of a signal, it is impossible to know when an event occurred and when it finished. Perhaps, if it is stationary signal, it will be not important thing. However, most interesting signals contain numerous non-stationary or transient characteristics: drift, trends, abrupt changes, and beginnings and ends of events. These characteristics are often the most important part of the signal. And, it makes MCSA based FFT digital signal processing to be not suitable enough to analysis current signal if we would like to know time information.

Development in digital signal processing have been resolved this problem. Short time Fourier transform (STFT) technique adapted of FFT has characteristic to analyze only a small section of the signal at a time - a technique called windowing signal. This technique is firstly introduced by Dennis Gabor (1946) as effort to resolve FFT limitation. STFT maps a signal into two dimensional functions of time and frequency.

STFT could make affiliation between time and frequency domain of a signal using both sequence of windowing and wide of windowing signal. So, in a time of windowing signal, we shall know what frequency occurred, and in the next of windowing, we also shall see if there is something changes about frequency compared before. Nevertheless, it will be dilemma to determine particular size for width of windowing. If we use big size for width of windowing, STFT will result good resolution of frequency, but poor for time resolution. It means that some frequencies of signal will be able to captured, but we do not know exactly when it is happen. However, if small size of windowing applied in STFT, we will get good resolution in time domain, but poor in frequency resolution

For this problem, next generation of digital signal processing after STFT can be used. It is wavelet transform. Thus, Wavelet transform represents the next logical step: a windowing technique with variable-sized regions. Wavelet analysis allows the use of long time intervals where more precise low-frequency information is required and shorter regions where high frequency information is required.

The main goal of this paper is to discuss the importance and reliability of wavelet transforms methods combined quadratic discriminant analysis for stator-current analysis to detect the faults in induction machines. The following section of this paper describes the most common faults in induction motors and their diagnosis using wavelet transform. Result of wavelet transform will be input in quadratic discriminant analysis to create groups of motor current based its condition. Novel approaches using wavelet transform and successfully quadratic discriminant analysis method are discussed in section III and IV with some experimental results. Finally, Section V presents some concluding remark.

\section{Wavelet Transform}

Wavelet transform is a transformation representing a signal to other domain. Wavelet transform is able to produce detail signal and smooth signal without time information losses. Wavelet transform is often used to process non stationary signal because of its capability to see the whole data of signal with desired resolution ${ }^{[13-17]}$.

Wavelet transforms works in space $\mathrm{L}^{2}(\mathrm{R})$. The letter of " $\mathrm{L}$ " signifies a Lebesque integral, the number of " 2 " denotes integral of square of function and " $R$ " states the independent variable of integration and $t$ is a number of whole real time. Thence $L^{2}(R)$ is a space of square integral able function, or for more clearly, it can be described as formula (1) follow:

$\int_{-\infty}^{\infty} f^{2}(t) d t<\infty$

It is mean that formality, if $f(\mathrm{t}) \in \mathrm{L}^{2}(\mathrm{R})$, then $f(\mathrm{t})$ must be able to be decomposition as linear combination of orthonormality basis function.

Wavelet transform is able to change signal resolution of original signal with scaling variable and then translate it from start to end of a signal. It is able to be described as formula (2) follow:

$F(\tau, s)=\frac{1}{\sqrt{s}} \int f(t) \Psi\left(\frac{t-\tau}{s}\right) ; s>0, \tau \in Z$

with $\tau$ is translation parameter, $\mathrm{s}$ is dilatation parameter, $f(t)$ is original signal, $\Psi$ is wavelet transform and $\mathrm{Z}$ is integer number. The formula (2) is also called as continuous wavelet transform. However, it is difficult to imagine that infinite signal can be transform with a transformation theorem. Therefore, a derivative of wavelet theorem, namely discrete wavelet transform is discovered to solve this problem. It can be made with sampling infinite signal into discrete signal. If continuous wavelet transform use integral transformation approach in signal processing, then discrete wavelet transform use multi resolution analysis approach to obtain desired signal.

Formula of decomposition signal $f(t)$ is define as formula (3). Signal can be represented by low frequency signal $c_{j}$ 
and high frequency signal $d_{j}$

$$
\begin{aligned}
\mathrm{f}(\mathrm{t})=\sum_{\mathrm{k}} \mathrm{c}_{\mathrm{j}}(\mathrm{k}) 2^{\frac{\mathrm{j}}{2}} \Phi\left(2^{\mathrm{j}} \mathrm{t}-\mathrm{k}\right)+ \\
\sum_{k} d_{j}(k) 2^{\frac{j}{2}} \psi\left(2^{j} t-k\right)
\end{aligned}
$$

Haar wavelet is one of wavelet type that widely known as simplest wavelet.. Father, $\phi$, and mother wavelet, $\psi$, in interval $\{0,2 \mathrm{~N}-1\}$, which $\mathrm{N}$ is integer number $\geq 1$. If value of $\mathrm{N}=1$, then, it can be described as follow:

$\psi=\left\{\begin{array}{cc}1 & \text { for } t\left[0, \frac{1}{2}\right] \\ -1 & \text { for } t\left[\frac{1}{2}, 1\right] \\ 0 & \text { oherwise }\end{array}\right.$

$\phi=\left\{\begin{array}{l}1 \text { for } 0<t<1 \\ 0, \text { otherwise }\end{array}\right.$

Formula (4) and (5), is also called as ' $d b l$ '. Commonly, Wavelet Doubechies is written as ' $d b N$ ', with $N$ is order of wavelet.

\section{Experiment Setup}

For purpose of this research, Experiment scale laboratory is performed to obtain spectrum of motor current. Three phase induction motor, $2 \mathrm{HP}, 4$ pole, 380/220 Volt, and 2.2/3.8 A is used in this experiment. Motor also has 36 slots and 61 winding of each slot. Stator winding of motor has been modified to represent number of winding of short circuit in motor as shown in Fig. 1. To get variation of short circuit case, selector switch is moved based on tapping of stator winding as shown in Table 1. Temporary short circuit is applied to this motor. Duration of temporary short circuit is $50 \mathrm{~ms}$ or 2.5 cycles. To get same time duration of each case, microcontroller and solid state relay (SSR) is used in this experiment. Phenomena of short circuit inter turn are captured by National Instrumentation data acquisition PXIe-5112. Current spectrum is recorded by $10 \mathrm{KHz}$ sampling frequency to capture fast transient current during starting and ending of short circuit. The experiment step is shown in Fig.2.

A total of 420 datasets was obtained. 330 datasets is used for training and other 90 datasets for testing. Number of shorted turn is used as variation each case of dataset as shown in Table 2.

\begin{tabular}{|c|c|c|c|}
\hline $\begin{array}{l}\text { Number } \\
\text { of cases }\end{array}$ & $\begin{array}{l}\text { Type of } \\
\text { Cases }\end{array}$ & Tapping & $\%$ of winding \\
\hline 40 & Normal & - & - \\
\hline 10 & \multirow{3}{*}{$\begin{array}{c}\text { Starting } \\
\text { fault }\end{array}$} & $0-5$ & 1,366 \\
\hline 10 & & $0-10$ & 2,732 \\
\hline 10 & & $0-25$ & 6,831 \\
\hline 10 & \multirow{3}{*}{$\begin{array}{c}\text { Steady } \\
\text { state fault }\end{array}$} & $1-25$ & 6,557 \\
\hline 10 & & $2-25$ & 6,284 \\
\hline 10 & & $3-25$ & 6,011 \\
\hline 10 & \multirow{2}{*}{$\begin{array}{c}\text { Ending } \\
\text { Fault }\end{array}$} & $5-25$ & 5,464 \\
\hline 10 & & $10-25$ & 4,098 \\
\hline
\end{tabular}

Table.1 Variation of shorted winding

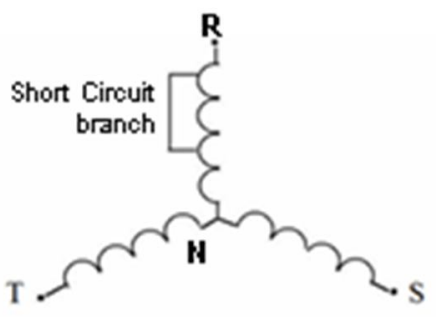

(a)

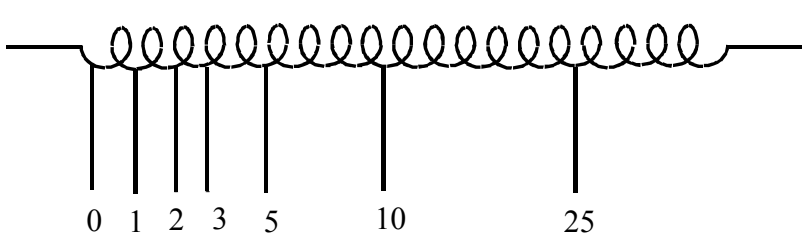

(b)

Fig. 1. (a) Wye winding of motor (b) Modification in Winding for short circuit tap

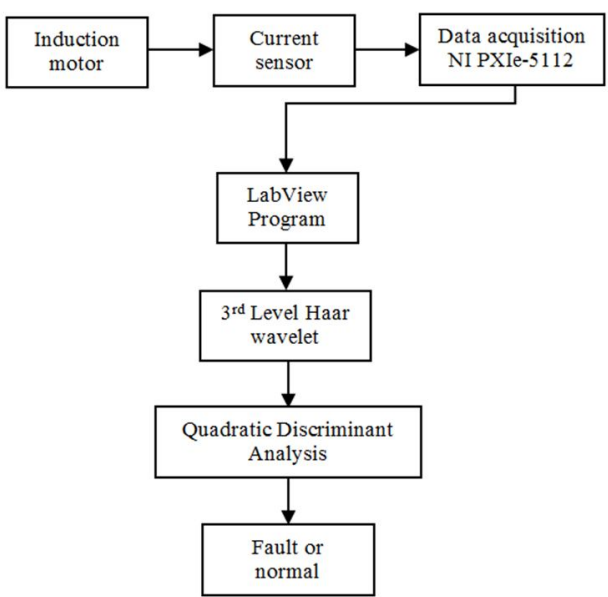

Fig. 2. Recording of short circuit phenomena 
Table 2. Motor current datasets

\begin{tabular}{|c|c|c|c|c|}
\hline \multirow{2}{*}{ Cases } & \multicolumn{2}{|c|}{ Training datasets } & \multicolumn{2}{c|}{ Testing datasets } \\
\cline { 2 - 5 } & $\begin{array}{c}\text { Number } \\
\text { of cases }\end{array}$ & $\begin{array}{c}\text { Variation } \\
\text { of cases }\end{array}$ & $\begin{array}{c}\text { Number } \\
\text { of cases }\end{array}$ & $\begin{array}{c}\text { Variation } \\
\text { of cases }\end{array}$ \\
\hline Normal & 120 & $\begin{array}{c}2,25 \mathrm{~A} ; \\
2,6 \mathrm{~A}\end{array}$ & 40 & $\begin{array}{c}2,25 \mathrm{~A} ; \\
2,6 \mathrm{~A}\end{array}$ \\
\hline $\begin{array}{c}\text { Starting } \\
\text { short } \\
\text { circuit }\end{array}$ & 105 & $\begin{array}{c}10 ; 15 ; 20 ; \\
22 ; 23 ; 24 ; \\
25\end{array}$ & 25 & $\begin{array}{c}10 ; 15 ; 20 ; 23 ; 24 ; \\
22 ;\end{array}$ \\
\hline $\begin{array}{c}\text { Ending } \\
\text { short } \\
\text { circuit }\end{array}$ & 105 & $\begin{array}{c}10 ; 15 ; 20 ; \\
22 ; 23 ; 24 ; \\
25\end{array}$ & 25 & $\begin{array}{c}22 ; 23 ; 24 ; \\
25\end{array}$ \\
\hline Total & 330 & & 90 & \\
\hline
\end{tabular}

\section{Experiment Result}

Current spectrum of induction motor during temporary short circuit is shown in Fig.3. Short circuit start at 0.055 $\mathrm{ms}$ and finish at $0.12 \mathrm{~ms}$, which means it has duration 65 ms. in this case, amplitude of short circuit current is 3.82 Ampere.

Fig. 4-6 shows high frequency of short circuit signal using first, second, and third level of wavelet analysis. As shown in those figure, third level wavelet transform results high frequency signal that shows us start and end of short circuit clearly.

Fig.7 shows quadratic of high frequency signal in third level wavelet transform. It can be seen that between starting and ending of fault have high signal. Fig. 8 shows energy level of faulty signal. It performs that during short circuit case there is enhancement of energy level. So, starting and ending of short circuit can be analyzed based on its energy level. The energy level is obtained using formula (6) which has period sampling $k$ is $10 \mathrm{~ms}$.

$e=\sum_{1}^{k}\left(d_{j}(k)\right)^{2}$

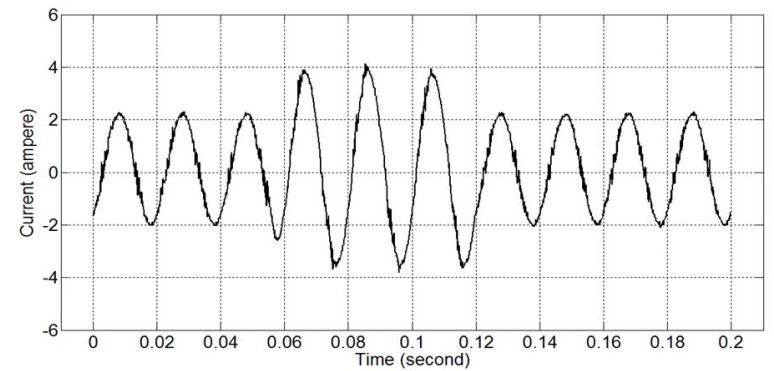

Fig. 3. Short circuit current of motor

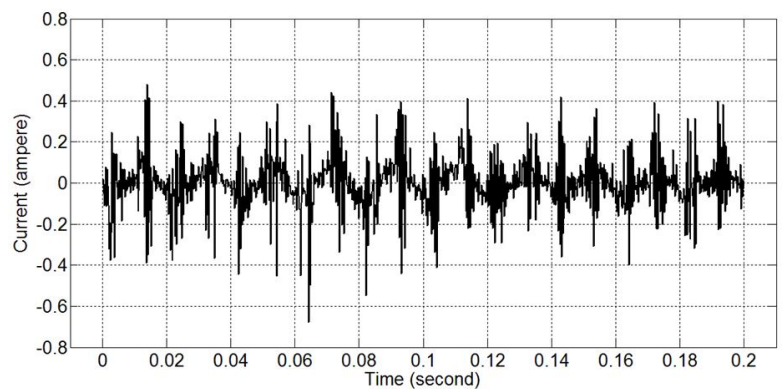

Fig. 4. High frequency signal using $1^{\text {st }}$ level of Haar wavelet

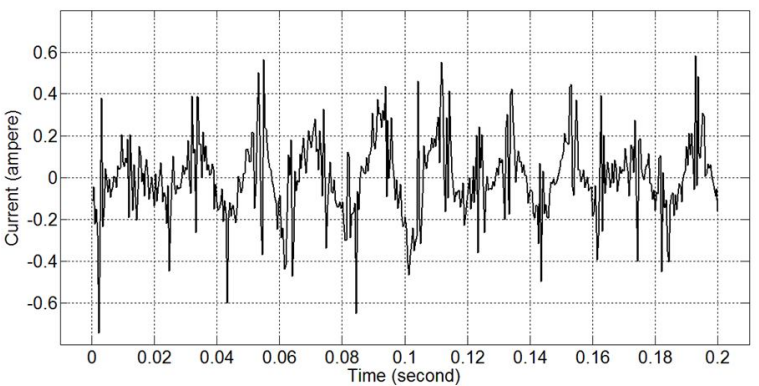

Fig. 5. High frequency signal using $2^{\text {nd }}$ level of Haar wavelet

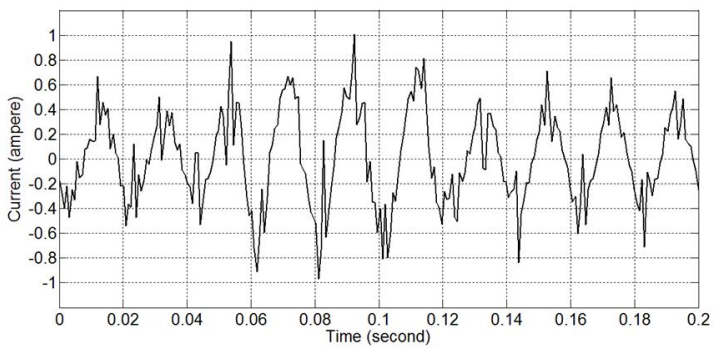

Fig. 6. High frequency signal using $3^{\text {rd }}$ level of Haar wavelet

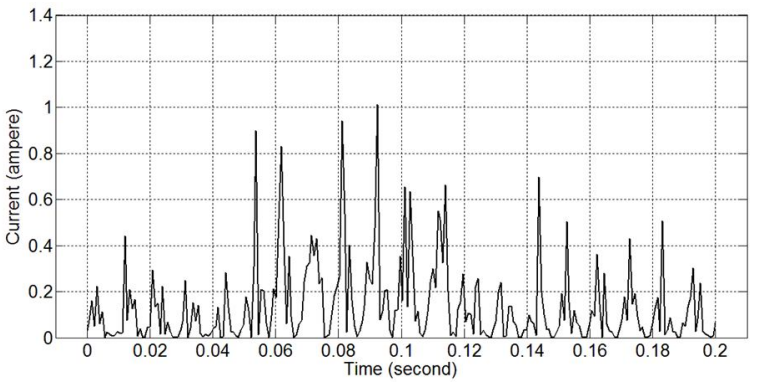

Fig. 7. High frequency signal square using $3^{\text {rd }}$ level of Haar

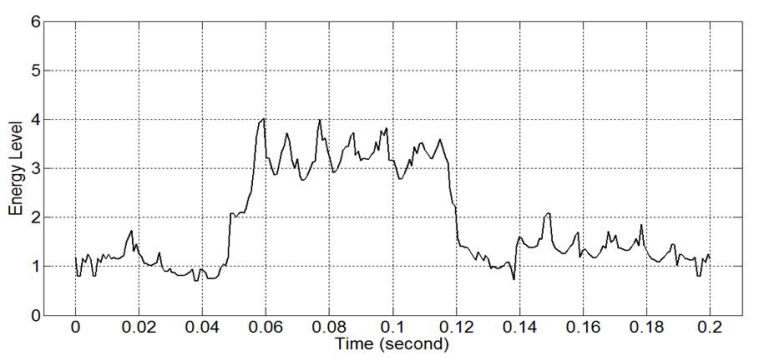

Fig. 8. Energy level 


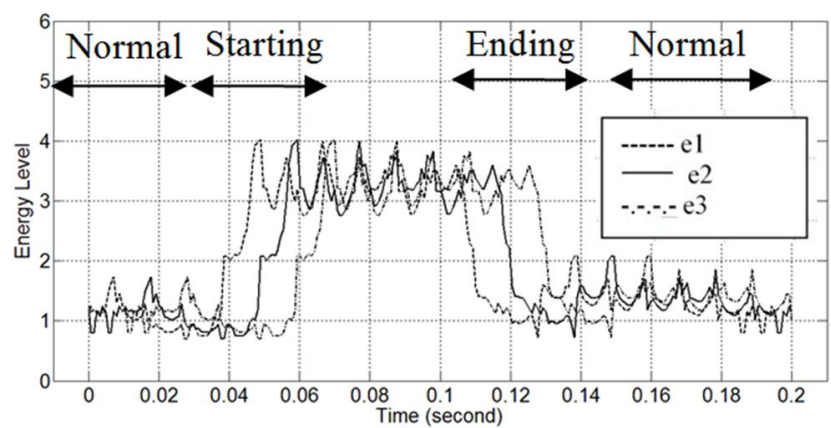

Fig. 9. Energy level of $e_{1}, e_{2}$, and $e_{3}$

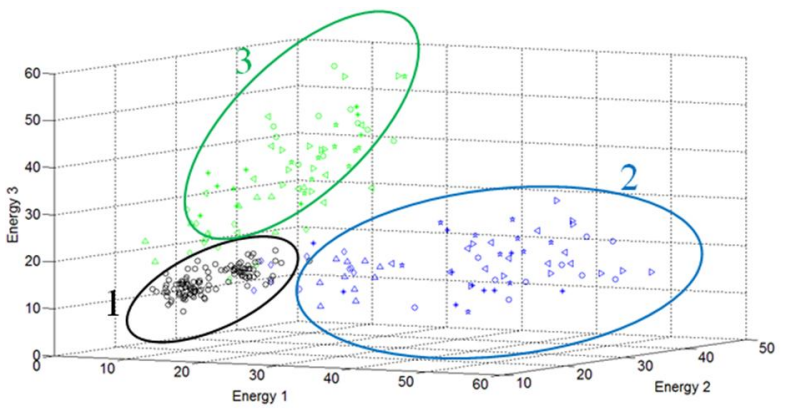

Fig. 10. Characteristic of motor current classification in 1) normal condition, 2) Starting fault and 3) Ending fault

Table 3. Energy level of current motor

\begin{tabular}{|c|c|c|c|}
\hline \multirow{2}{*}{ Cases } & \multicolumn{3}{|c|}{ Energy Level } \\
\cline { 2 - 4 } & $e_{1}$ & $e_{2}$ & $e_{3}$ \\
\hline Normal & Low & Low & Low \\
\hline Starting short circuit & High & Medium & Low \\
\hline Ending short circuit & Low & Medium & High \\
\hline
\end{tabular}

Table 4. Simulation output of training datasets using discriminant analysis

\begin{tabular}{|c|c|c|}
\hline Cases & $\begin{array}{c}\text { Accuracy of Linear } \\
\text { Discriminant } \\
\text { Analysis (\%) }\end{array}$ & $\begin{array}{c}\text { Accuracy of Quadratic } \\
\text { Discriminant } \\
\text { Analysis(\%) }\end{array}$ \\
\hline Normal & 100 & 100 \\
\hline Starting short circuit & 76.19 & 95.24 \\
\hline Ending short circuit & 80.95 & 90.48 \\
\hline
\end{tabular}

Table 5. Simulation output of testing datasets using discriminant analysis

\begin{tabular}{|c|c|c|}
\hline Cases & $\begin{array}{c}\text { Accuracy of Linear } \\
\text { Discriminant } \\
\text { Analysis (\%) }\end{array}$ & $\begin{array}{c}\text { Accuracy of Quadratic } \\
\text { Discriminant } \\
\text { Analysis(\%) }\end{array}$ \\
\hline Normal & 100 & 100 \\
\hline Starting short circuit & 84 & 96 \\
\hline Ending short circuit & 88 & 88 \\
\hline
\end{tabular}

Fig. 9 shows input variable $e_{1}, e_{2}$, and $e_{3}$. Actually, this variable is gotten from $e_{2}$ signal, but it is shifted by $10 \mathrm{~ms}$ to get $e_{1}$ and it is delayed by $10 \mathrm{~ms}$ to get $e_{3}$. Value of $e_{1}, e_{2}$, and $e_{3}$ will be used to analyze normal current, starting fault current and ending fault current. The characteristic of fault types also can figure out based on energy signal as shown in Fig. 10. Characteristic of $e_{1}, e_{2}$, and $e_{3}$ in normal current, starting fault current and ending fault current can be described in Table 3.

\section{Fault Classification based on Quadratic discriminant Analysis}

Normal current and fault current, especially starting and ending fault will be classified by discriminant analysis. Input of discriminant analysis is $e_{1}, e_{2}$, and $e_{3}$ of normal current, starting fault current and ending fault current. As comparison, linear discriminant analysis will be performing besides analyzed by quadratic discriminant analysis. Some datasets for training will be also evaluated using linear and quadratic discriminant analysis to investigate accuracy of this method. Then, 50 datasets for testing will be feed to discriminant analysis to evaluate robust of discriminant analysis. Result of discriminant analysis in some datasets for training can be seen in Table 4 .

Table 4 shows that quadratic discriminant analysis is more accurate to classify each of case. Quadratic discriminant analysis result accuracy is $100 \%$ for normal cases, $95.24 \%$ for starting fault cases which has single false detection data and $90.48 \%$ for ending fault cases which detection false data are two cases. Whereas, linear discriminant analysis results fitness $100 \%$ for normal current cases, $76.19 \%$ for starting fault cases which five false detection cases and $80.95 \%$ for ending fault cases which four false detection cases. For result of discriminant analysis in 50 datasets for testing, we can see in Table 5. Table 5 shows that quadratic discriminant analysis is more accurate to classify each of case. Quadratic discriminant analysis result accuracy is $100 \%$ for normal current cases, $96 \%$ for starting fault cases which single case false detection and $88 \%$ for ending fault cases or three cases false detection. Whereas, linear discriminant analysis results accuracy is $100 \%$ for normal current cases, $84 \%$ for starting fault cases or four false detection cases and $88 \%$ for ending fault cases or three fault detection cases. 


\section{Conclusions}

This paper describes capability of third level Haar wavelet transform combined with quadratic discriminant analysis to identification temporary fault in stator winding of induction motor. In this proposed method, detection based on starting and ending fault using energy level is presented. Energy level is obtained from high frequency signal of third level Haar wavelet transform. And then, Energy level of normal current, starting and ending fault current is used to input for quadratic discriminant analysis. Experimental setup is performed to get 420 datasets varied by number of shorted turn of stator winding of induction motor. 420 datasets is divided into 330 datasets for training and 90 datasets for testing. The result showed that quadratic discriminant analysis gives about $90 \%$ accuracy for classifying starting and ending fault current.

\section{References}

(1) W.T.Thomson, M. Fenger, "Case Histories of Current Signature Analysis to Detect Faults in Induction Motor Drives", Electrical Machines and Drives, IEMDC IEEE, Vol 3; pp 1459-1465, 2003.

(2) L.A. Pereira, Porto Alegre and D. da Silva Gazzana, "Motor current signature analysis and fuzzy logic applied to the diagnosis of short-circuit faults in induction motors," 31st Annual Conference of IEEE Industrial Electronics Society, 2005. IECON 2005. Vol. 1, no.1, pp. 6-10, 2005

(3) Xu Bo-qiang, Li He-ming and Sun Li-ling,"'Detection of stator winding inter-turn short circuit fault in induction motors," International Conference on Power System Technology,PowerCon 2004, Vol.2, pp.1005-1009 Nov. 2004.

(4) $\mathrm{Xu}$ Boqiang, Li Heming and Sun Liling, "Feature signal extraction of inter-turn short circuit fault in stator windings of induction motors," International Conference on Industrial Technology,IEEE ICIT '02, vol.1, pp.97-100, 2002..

(5) Osheiba, A.M. Darwish, H.A.; Taalab, A.-M.I.; Afify, A.A, "Transient modeling of stator winding inter-turn short-circuits in induction motors," Eleventh International Middle East Power Systems Conference, MEPCON 2006, vol. 2, pp. 643-647, 2006.

(6) R Beran, L., "Thermal effect of short-circuit current in low power induction motors," 13th Power Electronics and Motion Control Conference EPE-PEMC 2008, pp. 782-786, 2008

(7) Tong Liu, and Jin Huang, "A novel method for induction motors stator interturn short circuit fault diagnosis by wavelet packet analysis", Proceedings of the Eighth International Conference on Electrical Machines and Systems, ICEMS 2005, vol. 3 pp. 2254-2258, 2005.

(8) J. Y. Sang, C. Hao, P. C. Wang, "Diagnosis of Stator Winding Inter-Turn Circuit Faults in Induction Motors Based on Wavelet Packet Analysis and Neural Network", Advanced Materials Research, Vol. 529, pp. 37-42, 2012.

(9) W.T. Thomson, and R.J. Gilmore, "Motor Current Signature Analysis to Detect Faults in Induction Motor Drives - Fundamentals, Data Interpretation, and Industrial Case Histories," Proceedings of 32nd Turbomachinery Symposium 2003.

(10) M. E. H. Benbouzid, "A review of induction motors signature analysis as a medium for faults detection" IEEE Trans. Ind. Electron., vol. 47, no. 5, pp. 984-993, 2000.

(11) W. T. Thomson and M. Fenger, "Case histories of current signature analysis to detect faults in induction motor drives," Proceding IEEE IEMDC, Jun. 2003, vol. 3,pp. 1459-1465, 2003.

(12) Stephane Mallat," A Wavelet Tour of Signal Processing". Pour ma mere, Francine.October 9, 2008

(13) D.A. Asfani, Syafaruddin, M.H. Purnomo, T. Hiyama, "Wavelet- LDAneural network based short circuit occurrence detection in induction motor winding," IEEE International Symposium on Diagnostics for Electric Machines, Power Electronics \&Drives, SDEMPED 2011, pp.330-336, 2011.

(14) Gomes, Jonas. Velho, Luiz." From Fourier Analysis to Wavelets,".Instituto de Matematica Pura e Aplicada, IMPA. Rio de Janeiro, Brazil, 2001.

(15) G. Stephane. Mallat, "Multifrequency Channel Decomposition of Images and Wavelet Models", IEEE Transaction On Acoustics, Speech And Signal Processing. Vol.37. No.12, 1989.

(16) R.A. Johnson, D.W. Wichern, "Applied multivariate statistical analysis", 4th ed. Saddle River, NJ: Prentice-Hall, 1998.

(17) S. Girard Bouveyron and C. Schmid. "High Dimensional Discriminant Analysis". Communications in Statistics: Theory and Methods". Vol. 36, no. 14, pp. 2607-2623, 2007. 